\title{
Masquage des émotions et des attentes de soutien psychologique sur des forums d'entraide professionnelle
}

Masking emotions and expectations of psychological support on professional support forums

Magalie Prost, Béatrice Cahour et Françoise Détienne

\section{OpenEdition}

Journals

Édition électronique

URL : http://journals.openedition.org/activites/699

DOI : 10.4000/activites.699

ISSN : $1765-2723$

Éditeur

ARPACT - Association Recherches et Pratiques sur les ACTivités

\section{Référence électronique}

Magalie Prost, Béatrice Cahour et Françoise Détienne, « Masquage des émotions et des attentes de soutien psychologique sur des forums d'entraide professionnelle ", Activités [En ligne], 10-2 | Octobre 2013, mis en ligne le 15 octobre 2013, consulté le 01 mai 2019. URL : http://journals.openedition.org/ activites/699; DOI : 10.4000/activites.699

\section{(c) (†) $\ominus$}

Activités est mis à disposition selon les termes de la licence Creative Commons Attribution - Pas d'Utilisation Commerciale - Pas de Modification 4.0 International. 


\title{
Masquage des émotions et des attentes de soutien psychologique sur des forums d'entraide professionnelle
}

\author{
Magali Prost
}

Télécom ParisTech, Département SES, 46, rue Barrault - 75013 Paris, magaliprost@gmail.com

\section{Béatrice Cahour}

Télécom ParisTech-CNRS, Département SES, 46, rue Barrault, 75013 Paris, beatrice.cahour@ telecomparistech.fr

\section{Françoise Détienne}

Télécom ParisTech-CNRS, Département SES, 46, rue Barrault, 75013 Paris

francoise.detienne@telecom-paristech.fr

\begin{abstract}
Masking emotions and expectations of psychological support on professional support forums. The article focuses on the wellbeing of people at work and explores whether forums might be a new tool to improve it. The present study examines the subjective experience of forum participants during exchanges between education professionals, focusing on their expectations and emotional states. The challenge is to identify whether there are discrepancies between what the participants share in their messages and the experiences expressed during post-activity interviews. We carried out self-confrontation interviews followed by elicitation interviews with nine participants from the discussions we had previously analysed, based on written traces of these discussions. The results show that sharing experience (especially emotional) and personalized emotional support are the two most expected and satisfying types of support. The emotional dimension is very significant in the appreciation of support. However, in written discussions, we observed that participants tend to conceal specific emotional states that are difficult to communicate (shame, guilt, uneasiness). Expectations of emotional relief and psychological support are systematically hidden in written messages and are sometimes disguised as requests for advice. We observe that the reactants infer this type of expectation through emotional selfdisclosure. They then show this support through the type of support that they would expect for themselves or that has helped in the past. The results are discussed in relation to Walther's hyperpersonal communication theory.
\end{abstract}

\section{KEYWORDS}

forums for professionals, social support, self-confrontation interview, elicitation, masking, emotions, expectations, inferences

\section{1.- Introduction}

Les nouvelles formes d'organisation du travail tendent à réduire les espaces de discussion dédiés aux échanges entre professionnels et à entrainer une individualisation (Dejours, 
1993). Les «espaces de délibération » où se discutent les réajustements sont des espaces informels qui sont déconstruits par la performance comme unique critère d'évaluation. Ces mutations peuvent mettre les professionnels dans des situations d'isolement professionnel et de souffrance au travail (Dejours, 1998 ; Marc, Grosjean, \& Marsella 2011). Le bien-être au travail est une préoccupation de plus en plus investie au regard des drames qui se produisent dans diverses entreprises. L'ergonomie s'empare de ces questions et s'intéresse aux liens entre les formes de management et le bien-être au travail (Grosjean, \& Ribert-Van de Weerdt, 2005) et à la manière dont les individus trouvent des ressources et s'adaptent aux situations de travail difficiles (Ponnelle, \& Lancry, 2002).

Les évolutions technologiques offrent aux professionnels une possibilité de construire de nouveaux espaces sociaux pour parler de leurs difficultés et peut-être pallier certaines d'entre elles. Les individus peuvent interagir au travers de «communautés en ligne » (Proulx, \& Latzko-Toth, 2000). Ces communautés, dont les membres se retrouvent sur des plateformes dédiées (e.g. forums de discussion) autour d'un intérêt commun, ont des objectifs divers comme échanger du soutien social (Malik, \& Couslon, 2008; Pfeil, \& Zaphiris, 2007) ou construire des connaissances (Babinski, \& Jones, 2001; Chanier, \& Cartier, 2006) dans la lignée des études sur les communautés de pratique (Wenger, 1998). Au sein des communautés d'entraide virtuelles, les participants font part de leurs expériences notamment émotionnelles, ce qui renvoie au phénomène de partage social des émotions mis en évidence par Rimé (2005). Généralement, les individus ont tendance à exprimer leurs émotions, positives ou négatives, aux personnes intimes telles que la famille, les amis ou encore les collègues en situations professionnelles. Or, sur les forums les participants ne se connaissent pas a priori puisque les échanges se font sous couvert d'anonymat. Se pose alors la question de ce qui motive les participants à y participer, de la qualité de l'aide apportée sur ces forums et de ce qu'ils en tirent en termes de bien-être au travail.

L'étude présentée dans cet article fait partie d'un travail plus large sur les apports des forums de professionnels en termes de bien-être et d'acquisition de compétences sur la pratique (Prost, 2012). L'article vise à explorer le vécu subjectif de ceux qui participent aux forums dédiés aux professionnels, en se centrant sur les attentes, l'état émotionnel et l'évaluation du soutien émotionnel. La question est de savoir si les participants expriment des attentes et des émotions lors des interactions sur le forum conforment à celles qu'ils déclarent ressentir. Pour ce faire, nous aurons recours à une méthodologie originale qui consiste à confronter ce qui est socialement exprimé dans les discussions écrites et ce qui est de l'ordre du vécu de ces échanges exprimé en entretien afin de mettre à jour d'éventuels décalages.

L'article présentera, dans un premier temps, un état de l'art sur la communication médiatisée par ordinateur et plus spécifiquement la communication émotionnelle sur les forums; dans un second temps, les objectifs de l'étude et la méthodologie déployée seront précisés ; et enfin les résultats seront présentés et discutés au regard de la littérature.

\section{2.- État de l'art sur les émotions dans la communication médiatisée par ordinateur}

Il existe aujourd'hui deux théories dominantes sur la communication médiatisée par ordinateur que nous présenterons succinctement avant d'apporter des éclairages sur la façon dont ont été traitées les manifestations émotionnelles et leur interprétation dans les communications médiatisées, notamment sur les forums.

\section{1.- La communication médiatisée : deux théories opposées}

Un consensus se dégage au sein de la littérature pour mettre en avant deux caractéristiques principales responsables de transformation de la communication émotionnelle en Communication Ecrite Médiatisée par Ordinateur (CEMO) : l'anonymat et l'absence de comportements non verbaux. Toutefois, les auteurs se distinguent dans l'interprétation des conséquences de ces deux caractéristiques. Deux théories défendent des positions 
contradictoires : la théorie de la réduction des indices sociaux en déduit des conséquences négatives pour la qualité de l'échange social (Sproull, \& Kiesler, 1986) et la théorie de la communication hyperpersonnelle considère au contraire que les impacts en sont bénéfiques (Walther, 1996).

La première théorie stipule que l'anonymat et l'absence du canal non verbal entraînent les individus à centrer leurs échanges sur la dimension informationnelle au détriment de la dimension socio-émotionnelle. Les normes et les valeurs en vigueur en CEMO seraient responsables des comportements asociaux ou non réglementés observés en situation médiatisée (pour revue voir Derks, Fischer et Bos, 2007). La situation de CEMO entraverait l'expression et la compréhension des états mentaux (Fisher-Lokou, Guéguen, \& Lépy, 2004 ; Hebert, \& Vorauer, 2003), ce qui entrainerait des émotions négatives (Kato, Kato, \& Akahori, 2007) et une réticence à écrire (Zhang, Qu, Cody, \& Yuling, 2010). Les individus surestimeraient leur habileté à communiquer par email par manque d'anticipation des interprétations et de projection dans d'autres perspectives (Kruger, \& Epley, 2005).

Les conclusions de ces différentes études étayent la théorie du «paradoxe d'Internet» (Internet paradox) développée par Kraut, Patterson, Lundmark, Kiesler, Mukophadhyay et Scherlis (1998), selon laquelle les «technologies sociales», principalement utilisées pour l'interaction interpersonnelle, augmentent l'isolement social et diminuent le bien-être psychologique des utilisateurs. Selon Moody (2001), limiter les interactions en face à face peut entraîner de la solitude en dépit du sentiment de forte connexion avec le monde social. Les résultats des études qui s'inscrivent dans la perspective de la réduction des indices sociaux portent essentiellement sur la compréhension des états mentaux et ne traitent pas des manifestations émotionnelles qu'ils jugent réduites

La seconde théorie stipule au contraire que la communication en CEMO favorise l'expression émotionnelle (Derks et al., 2007). L'anonymat et la suppression des distances permettent un dévoilement de soi plus important et plus spontané que dans les relations non médiatisées (Barak, \& Gluck-Ofri, 2007 ; Jiang, Bazarova, \& Hancock, 2011 ; Lewkowicz, Marcoccia, Atifi, Bénel, Gaglio et al., 2008 ; Wright, \& Bell, 2003). La CEMO favoriserait le dévoilement des expériences émotionnelles négatives puisque leur exposition est moins menaçante en situation d'anonymat (Walther, \& Boyd, 2002). Caplan et Turner (2007) proposent la «théorie de la communication en ligne réconfortante» (online comforting communication) dans laquelle les caractéristiques du média aideraient à améliorer le bienêtre et à diminuer la détresse émotionnelle. Les participants réussiraient à établir des relations similaires, voire plus intimes, en CEMO qu'en face à face (Hian, Chuan, \& Trevor, 2004 ; Pfeil, Zaphiris, \& Wilson, 2009 ; Walther, 1996).

La perspective de Walther (1996) apparaît intéressante en ce qu'elle ne se limite pas aux caractéristiques du média comme seul déterminant des conduites observées sur Internet, mais vise à décrire les stratégies de manifestations émotionnelles des participants. Les travaux présentés dans la section suivante mettent l'accent sur les stratégies que les participants utilisent pour manifester leurs émotions.

\section{2.- Le dévoilement de soi comme manifestation de ses émotions en CEMO}

L'absence des signaux non verbaux en CEMO est palliée par davantage de descriptions explicites des états émotionnels, les individus adaptant leur contenu langagier (Derks et al., 2007). Le dévoilement de soi («self-disclosure») est le procédé discursif le plus étudié des études sur la communication émotionnelle en CEMO. Ces études s'intéressent principalement à des communautés virtuelles de patients ou de personnes âgées et peu à des communautés virtuelles de professionnels. Le dévoilement de soi est généralement défini comme l'ensemble des informations que donne une personne à propos d'elle-même à d'autres personnes. Cette définition très large est opérationnalisée de manière différente dans les études (comptage du nombre de «je», du nombre de mots du message, analyse de 
contenu qualitative, etc.). Le dévoilement de soi est un élément caractéristique des communautés de soutien en ligne (Barak, \& Gluck-Ofri, 2007 ; Preece, \& Ghozati, 2001) qui couvre plusieurs fonctions (Tichon, \& Shapiro, 2003) :

1. Demander du soutien de manière implicite. Pfeil et Zaphiris (2007) observent, à l'aide d'une analyse qualitative des messages, que les participants demandent rarement directement de l'aide ou du soutien ( $2 \%$ dans leur étude). Ils ont tendance, pour déclencher une réponse empathique, à faire un récit de leurs préoccupations plutôt que de poser directement une question (ibid., Preece, 1999).

2. Donner du soutien. Le réactant peut démontrer à son interlocuteur, à travers le dévoilement d'une expérience vécue, qu'une solution au problème est envisageable. Les participants préfèrent dire ce qu'ils feraient ou ont fait dans une situation similaire plutôt que de donner des conseils directs et explicites (Pfeil et al., 2009; Wright, 2000). Ils ont ainsi tendance à déguiser un conseil en partage d'expérience.

Le dévoilement de soi couvre également d'autres fonctions comme permettre aux participants de procéder à une comparaison sociale qui le plus souvent est source de réassurance (Walther, \& Boyd, 2002). Se dévoiler à des personnes qui vivent une situation stressante similaire permet de valider, normaliser l'expérience, réduire l'isolement émotionnel et de créer un sentiment d'appartenance (Helgeson, \& Gottlied, 2000). Malik et Coulson (2008) montrent que ce partage de vécus similaires peut aider les participants à prendre des décisions (e.g. sur les traitements médicaux).

Les études présentées dans cette section montrent que les participants aux communautés d'entraide virtuelles font part de leurs ressentis à travers le dévoilement de leurs expériences. Toutefois, elles ne permettent pas de savoir si les émotions qu'ils manifestent dans les échanges correspondent à leurs émotions vécues. La section suivante s'attache à présenter la compréhension des manifestations émotionnelles en CEMO et le phénomène de masquage des émotions.

\section{3.- Compréhension des manifestations émotionnelles en CEMO et masquage des émotions}

Les travaux sur la communication émotionnelle en CEMO s'attachent davantage à décrire les stratégies mises en place pour la communication des émotions que la compréhension de ces manifestations, sauf à travers la question de la dimension intime des échanges (Derks et al., 2007). Or, "le fait de manifester ses émotions ne garantit pas que le partenaire puisse les interpréter de façon pertinente »; par cette phrase Gauducheau (2008, p. 309) pointe un manque évident des études dans la mouvance de Walther (1996). Les travaux de Cosnier (1994) montrent que les émotions peuvent être masquées, camouflées, censurées en cours d'interactions dans les situations réelles. Bien que le partage des émotions soit une tendance caractéristique de l'épisode émotionnel (Rimé, 2005), certaines émotions telles que la honte et la culpabilité sont difficilement communicables en face à face (Finkenauer, \& Rimé, 1998) et l'on peut se demander si cette censure est levée par l'anonymat de la CEMO. Lipianski (1992) parle de «censure sociale », ou "masquage » pour faire référence à la censure volontaire et consciente qu'exerce l'individu. Cahour (2000) distingue également les émotions censurées de manière inconsciente qui ont trait à des processus de refoulement et de défenses psychiques; elle parle dans ce cas de censure «psychique » ou « inconsciente». Les motifs de la censure sociale peuvent être liés à la protection des faces afin d'assurer le bon déroulement des interactions (Goffman, 1974). L'individu, dans un autre cadre social comme l'entretien de recherche, peut être en mesure d'exprimer un ressenti masqué par ailleurs (Cahour, 2000). Dans la suite de cet article, nous parlerons de «masquage » pour faire référence tant aux masquages conscients que non conscients et préciserons lorsque nous voudrons souligner le caractère volontaire ou involontaire du masquage.

Du côté de la réception des messages, les quelques études qui se sont penchées sur la question de l'intelligibilité des émotions se sont centrées sur la compréhension des smileys 
(Walther, \& D'Addario, 2001) et ont montré la complexité du processus de compréhension des émotions manifestées dans un message (Marcoccia, \& Gauducheau, 2007). Les individus intègrent différentes informations pour décrypter le message (émoticônes, valeur expressive du message, valence des émotions). Le lecteur a des doutes sur la sincérité de la joie exprimée lorsque le locuteur utilise différents procédés expressifs.

Ce tour d'horizon des travaux sur la communication émotionnelle en situation médiatisée montre que nous savons peu de choses sur les éventuels phénomènes de masquage opérés par les participants au niveau de leurs émotions ainsi que sur la compréhension des manifestations émotionnelles. La section suivante présente les objectifs et le terrain de l'étude, à savoir deux forums de discussion entre professionnels de l'éducation.

\section{3.- Une étude des échanges entre professionnels sur des forums dédiés}

L'article s'intéresse à la communication émotionnelle dans les forums d'échanges entre professionnels et cherche plus spécifiquement à savoir si les participants ont recours à du masquage de leurs émotions et de leurs attentes de soutien psychologique. L'étude globale dans laquelle se situe le travail présenté ici a montré un résultat contradictoire entre, d'une part, les déclarations des participants aux forums professionnels via un questionnaire en ligne, qui expriment des motivations liées à la recherche de soutien psychologique, et l'analyse de contenu des échanges, qui met en évidence une dynamique interactionnelle de soutien psychologique; et d'autre part, l'absence de demandes de soutien explicites de ce type dans les discussions analysées. Ainsi, en nous basant sur ces résultats et sur ceux des études de Preece (1999) et Pfeil et Zaphiris (2007), nous postulons que les professionnels étudiés ne demandent pas explicitement du soutien émotionnel, mais que ce type de soutien est implicitement attendu. Nous cherchons également à savoir si les autres participants comprennent ces éventuelles attentes masquées et le cas échéant, sur quels éléments ils se basent pour faire cette inférence.

\section{1.- Contexte}

Les participants que nous avons interviewés se rendent sur deux forums dédiés à des professionnels. Le premier, lesocial.fr, est un site internet créé en 1996 qui regroupe dix-neuf forums correspondant chacun à un métier du secteur social et médico-social. Les cibles du site sont les professionnels en exercice ainsi que les personnes en formation. Il est présenté comme le premier site du secteur social et médico-social en France en terme de fréquentation et d'activité sur les différents forums (27059 visites / jour en octobre 2011). Il représente une source importante d'informations pour les professionnels de ce domaine (emploi, formation, actualité, législation, etc.). L'administrateur du site nous a autorisées à contacter les participants du forum «éducateurs spécialisés». Le second forum, enseignants-duprimaire.com (EDP), a été créé en 1998 par un jeune enseignant en manque de formation et d'informations, et est le plus important site Internet dédié aux professeurs des écoles (119367 membres inscrits et 98138 visites uniques en octobre 2011). La partie «forum » du site regroupe plusieurs sous-forums classés par thèmes (e.g. "l'école primaire», «le français »).

L'étude générale dans laquelle se situe l'étude présentée dans cet article (voir Prost, 2012) a montré, à travers un questionnaire en ligne et des entretiens, que les motivations des participants de ces deux forums ont trait à l'amélioration de la pratique professionnelle, à la résolution de problème (prise de décision et prise de distance), au soutien psychologique, à l'utilisation du forum comme palliatif au manque de formation et de soutien sur le lieu de travail. Les discussions sont principalement axées sur le thème de la pédagogie. Cette même recherche a montré, par une analyse de contenu qualitative des messages, que la moitié des messages des initiateurs contiennent des émotions négatives explicites dont les plus fréquentes sont liées au doute et à l'expression d'une difficulté suivie d'émotions liées au 
stress et à l'angoisse. Ceci tend à montrer que les participants font part dans les échanges, de situations problématiques pour eux.

\section{2.- Méthodologie}

Afin de répondre aux objectifs de l'étude, à savoir identifier les phénomènes de masquage des émotions et attentes et étudier le processus d'inférence, nous avons conduit neuf entretiens avec des participants ayant le statut d'initiateurs et de réactants. Les entretiens ont été basés sur la trace de leur activité, à savoir des discussions auxquelles ils ont participé. Les étapes de ce travail sont détaillées dans les sections suivantes.

\subsubsection{Prise de contact avec des participants et sélection des discussions-support}

La prise de contact avec des participants aux forums a été difficile à mettre en place, mais nous avons quand même pu réaliser des entretiens avec neuf personnes. Différentes stratégies ont été adoptées, notamment l'observation participante, l'envoi d'un questionnaire en ligne et la recommandation par la première personne interviewée. Une fois qu'un participant avait accepté l'entretien, nous cherchions dans l'historique de ses messages, si possible, une discussion pour laquelle il avait été l'initiateur et une autre où il avait été réactant. Ceci permet d'avoir des éléments de compréhension du vécu des personnes en demande d'aide et des personnes qui proposent de l'aide.

$\mathrm{Au}$ total, neuf entretiens ont été menés. L'ensemble des personnes interrogées sont des femmes entre 25 et 45 ans, huit fréquentant EDP et une le social. Le tableau 1 présente une synthèse des discussions, du statut des interviewées et du laps de temps entre les échanges écrits sur forums et l'entretien.

\begin{tabular}{|c|c|c|c|}
\hline Sujet de la discussion & $\begin{array}{l}\text { Durée de la } \\
\text { discussion }+ \text { nb } \\
\text { de messages }\end{array}$ & $\begin{array}{l}\text { Laps de temps } \\
\text { entre écrits et } \\
\text { entretien }\end{array}$ & $\begin{array}{c}\text { Statut des } \\
\text { participantes } \\
\text { interviewées }\end{array}$ \\
\hline Dysfonctionnement institutionnel & $\begin{array}{c}6 \text { jours } \\
9 \text { messages }\end{array}$ & 12 mois & Initiatrice \\
\hline Élève qui n'aime pas l'école & $\begin{array}{c}1,5 \text { jour } \\
6 \text { messages }\end{array}$ & 9 mois & Initiatrice \\
\hline Méthode de lecture & $\begin{array}{c}3 \text { jours } \\
34 \text { messages }\end{array}$ & 10 jours & Réactante \\
\hline Élève qui pleure tous les matins & $\begin{array}{c}4 \text { jours } \\
11 \text { messages }\end{array}$ & 7 mois & Réactante \\
\hline Agression par un élève & $\begin{array}{c}11 \text { jours } \\
61 \text { messages }\end{array}$ & $\begin{array}{c}11 \text { jours } \\
35 \text { jours } \\
2 \text { mois } \\
1 \text { mois et demi }\end{array}$ & $\begin{array}{l}\text { Initiatrice } \\
\text { Réactante } \\
\text { Réactante }\end{array}$ \\
\hline Gestion d'une classe difficile & $\begin{array}{c}1 \text { jour } \\
6 \text { messages }\end{array}$ & 32 mois & Initiatrice \\
\hline $\begin{array}{l}\text { N'arrive pas à faire tout son } \\
\text { programme et ne sait pas sur quoi } \\
\text { insister }\end{array}$ & $\begin{array}{c}2 \text { jours } \\
17 \text { messages }\end{array}$ & 2 mois & Initiatrice \\
\hline Lassitude de son métier & $\begin{array}{c}4 \text { jours } \\
23 \text { messages } \\
\end{array}$ & 2 mois et demi & Réactante \\
\hline $\begin{array}{c}\text { Problèmes relationnels avec ses } \\
\text { collègues et la hiérarchie à cause } \\
\text { de ses absences }\end{array}$ & $\begin{array}{c}2 \text { heures } \\
49 \text { messages }\end{array}$ & 12 mois & Initiatrice \\
\hline Gestion d'une classe difficile & $\begin{array}{c}4 \text { jours } \\
9 \text { messages }\end{array}$ & 21 mois & Initiatrice \\
\hline Livret scolaire à améliorer & $\begin{array}{c}15 \text { jours } \\
34 \text { messages }\end{array}$ & 5 jours & Initiatrice \\
\hline
\end{tabular}

Tableau 1 : Présentation des discussions et du statut des interviewées

Table 1: Presentation of discussions and of interviewee status 
Le délai parfois important entre le moment de l'écrit (de 5 jours à 30 mois) constitue une difficulté méthodologique. Afin de la limiter, nous avons eu recours à différentes précautions méthodologiques que nous développerons dans la partie suivante.

\subsection{2.- Recueil de données : entretiens d'auto-confrontation avec relances d'entretiens d'explicitation}

Les entretiens, d'une durée de deux à trois heures, ont été intégralement filmés. La caméra était focalisée sur les traces écrites de la discussion afin de faciliter, lors de la retranscription, la mise en correspondance entre les verbalisations du participant et les moments de la discussion dont il était question. L'interviewée était invitée à découvrir la discussion au fur à et à mesure de sa lecture.

Les entretiens se sont déroulés en deux phases : une première phase de familiarisation avec la participante où l'intérêt portait sur son parcours professionnel, son travail, sa pratique du forum, ses habitudes, ses attentes et motivations à participer au forum. La seconde phase était dédiée à l'entretien d'auto-confrontation où nous laissions la participante relire la discussion et la commenter quand elle le souhaitait. Nous la relancions à certains moments, notamment lorsque des émotions étaient exprimées dans les messages.

Afin de limiter le délai parfois important entre le moment des échanges écrits et celui de l'entretien, il était proposé aux participantes de verbaliser leur vécu à partir des traces de leur activité et en utilisant des relances d'entretien d'explicitation (EdE) (Cahour, 2012). Les relances d'EdE favorisent la mise en évocation ${ }^{1}$ du sujet, ce qui limite le risque de reconstruction et permet de recueillir des données plus proches de l'expérience vécue du sujet (Vermersch, 1994). Nous citerons ici les deux types de relances que nous avons principalement utilisées lors des entretiens : les relances «vides de contenu » qui «doivent rester les plus ouvertes possible de sorte que l'expérience du sujet, quelle que soit sa nature, puisse s'y loger » (Cahour, 2012, p. 26) ; les relances en écho qui consistent à reprendre de manière interrogative un mot ou un groupe de mots venant d'être prononcé. Les indices visuels (décrochement du regard, gestes «mimes »), auditifs (diminution du flux de paroles et de l'intensité de la voix) et linguistiques (temps utilisés), établis par Vermersch (1994) puis Cahour (2012), ont permis de déceler des moments où la personne n'était pas en évocation.

Enfin, pour nous assurer de l'évocation de la participante, nous lui demandions régulièrement si ses propos étaient en lien avec ce qu'elle pensait/ressentait au moment de l'interaction écrite ou au moment de l'entretien. Cahour (ibid.) explique que l'évocation peut être complète (le sujet est ré-immergé dans la situation) ou partielle (le sujet se rappelle de certains éléments sans toutefois revivre complètement la situation). Dans l'étude, toutes les participantes (sauf une) ont été en évocation sur une partie ou sur l'ensemble de la discussion. Le laps de temps entre le moment des échanges et l'entretien ne semble pas être la principale cause de l'évocation partielle. On remarque que pour les trois discussions pour lesquelles le délai entre écrits et entretien est le plus long (12, 21 et 30 mois), les participantes avaient de bons souvenirs de l'épisode relaté et ont été en évocation durant l'entretien. L'évocation semble ainsi avoir été facilitée par la saillance émotionnelle de l'épisode vécu tel que décrit par Wagennar (1986). L'une des neuf participantes a par contre rationalisé son expérience tout au long de l'entretien qui portait sur la discussion qu'elle avait initiée. Des indices discursifs tels que «je pense que », «il me semble», «je crois» ont permis de comprendre qu'elle n'était pas en évocation. Cela est peut-être dû au fait que nous avions réalisé un entretien avec l'une des collègues de son établissement avec laquelle les relations sont parfois difficiles. La participante était peut-être réticente à se livrer par crainte que nous puissions en parler à sa collègue. Sur la base de ces critères, nous avons

1 Il s'agit d'aider, avec des techniques de relances particulières, le sujet à se mettre en évocation de la situation qu'il a vécue, c'est-à-dire à en recontacter un souvenir vivace. 
exclu cette discussion de l'analyse.

\subsection{3.- Analyse des données}

Dans la lignée des travaux de Cahour, Brassac, Vermersch, Bouraouis, Pachoud et Salembier (2007) et Cahour (2012), nous avons cherché à mettre en évidence des décalages en confrontant des données observables et le point de vue du sujet. Le traitement des données a suivi différentes étapes: dans un premier temps, les entretiens ont été intégralement retranscrits. Nous avons ensuite distingué au sein des verbalisations, celles qui ont trait au moment des échanges écrits sur le forum et celles correspondant à une analyse réflexive de cette activité. Cette précaution méthodologique a été facilitée, comme expliqué précédemment, par l'observation des indices linguistiques (e.g. " je pense que », « je trouve que », « en général », etc.) et par les sollicitations à l'égard des participantes afin de nous assurer de la mise en évocation. Puis, nous avons mis en regard ce qui était échangé lors des discussions en ligne et ce qui était dit pendant les entretiens sur le vécu de cet échange. Enfin, nous avons repéré dans les discussions écrites et les entretiens, les séquences où les participantes expriment leurs émotions, leurs attentes et leur compréhension des attentes de soutien psychologique afin d'observer la présence ou non de décalages.

Nous traiterons, dans cet article, des émotions exprimées par le participant lors de l'interaction sur le forum, autrement dit celles qu'il ressent lorsqu'il écrit le message. Notons qu'il est difficile, d'un point de vue méthodologique, de faire la distinction entre ces émotions et celles qu'il a ressenti dans la situation qu'il relate. Généralement, l'initiateur partage une expérience qu'il a vécue quelques heures auparavant, ce qui peut laisser penser que les émotions ressenties dans ces deux moments (situation relatée et moment des échanges sur le forum) sont de même nature et nous ne chercherons pas à établir cette distinction dans cet article.

\section{4.- Résultats}

L'analyse de nos données permet de mettre en avant trois types de résultats : les premiers sont relatifs aux modes de soutien particulièrement appréciés par les participantes, à savoir le partage d'expérience et le soutien émotionnel personnalisé ; les deuxièmes à la mise en évidence du masquage de certaines émotions par les participantes; et les troisièmes aux attentes de soutien psychologique sont d'une part masquées par les initiatrices et d'autre part, inférées par les réactantes.

\section{1.- Le partage d'expérience et le soutien émotionnel personnalisé comme éléments du soutien les plus appréciés}

Le partage d'expérience est un mode de soutien attendu par l'ensemble des participantes. L'expérience vécue a une légitimité pour les participantes qui n'est pas retrouvée pour les autres modes de soutien "Il y a cette notion de partager et c'est vrai que c'est ça moi que je recherche, il y en a qui donnent un avis sans avoir vécu la chose, mais c'est différent, c'est bien d'avoir un avis de quelqu'un qui a rencontré les mêmes difficultés. ». Pour l'ensemble des participantes interviewées, le partage d'expérience est gage de la connaissance de la situation par l'interlocuteur et apporte de la crédibilité au conseil «ça a plus de poids effectivement si la personne a vécu les choses et trouver des solutions. Parce que sinon je me dis que la personne elle me propose des choses (...), mais elle risque d'être à côté de la plaque et ne pas vraiment comprendre les soucis. Je fais plus attention aux personnes qui ont déjà vécu la même chose. »

Ce mode de soutien a des fonctions différentes selon le contexte et les participantes :

- Il peut être la source d'amélioration de sa pratique «Ça m'a semblé ambitieux et difficile, mais à force de lire les interventions, je me suis lancée là-dedans [dans une nouvelle méthode de lecture], mais je n'aurais jamais eu le courage de le faire s'il n'y 
avait pas eu différentes personnes, différents retours, ça a vraiment changé ma pratique professionnelle».

- Il peut aider à la construction de connaissances en alimentant le référentiel des situations connues «Je voulais plutôt savoir ce qui s'était passé et voir ce que je serais censée faire »

- Il peut aider à prendre une décision au travers d'un partage d'expérience émotionnelle négative «ça m'a permis aussi de me dire :'je ne veux pas de ça, (...) je ne veux pas de ce genre de stress au travail, de ce genre de maladie', ça m'a aidé à prendre ma décision [démissionner] »

- Il peut favoriser la proximité affective dans le cas de partage d'expérience émotionnelle similaire "Ça m'a vachement touché parce que le gars il explique qu'il est allé jusqu'à être hospitalisé (...) ça me rapprochait de lui en fait, c'était ça, parce que moi je m'étais livrée aussi dans mon message, du coup, je trouvais que ça me rapprochait de lui. »

- Il peut être la source de réassurance par la comparaison sociale «Je me souviens qu'on m'avait dit que certaines vivaient la même chose et que c'était difficile et ça m'avait aidé de l'entendre ». Le fait de voir que d'autres personnes vivent des situations similaires et ont le même type de ressenti permet de réduire le sentiment de solitude et prendre de la distance.

On observe que le soutien apporté par le partage d'expérience est souvent traduit sur le plan émotionnel.

Le soutien émotionnel est une autre attente partagée par toutes les interviewées quand elles sont en position d'initiatrices. Les résultats montrent que le soutien émotionnel personnalisé satisfait davantage les participantes que le soutien émotionnel stéréotypé (marques traditionnelles de soutien de type «bon courage», «prends soin de toi », «tiens bon »). Les manifestations stéréotypées peuvent apparaître assez peu efficaces comme en témoignent les exemples du tableau 2.

\begin{tabular}{|l|l|l|}
\hline $\begin{array}{l}\text { Type de soutien } \\
\text { émotionnel }\end{array}$ & Extrait du message du réactant & Réactions de l'initiatrice en entretien \\
\hline Stéréotypé & $\begin{array}{l}\text { Je compatis complètement à ta } \\
\text { situation }\end{array}$ & $\begin{array}{l}\text { j'ai envie de dire tant mieux. (...) ça ne me } \\
\text { touche pas plus que ça parce que je trouve } \\
\text { que c'est une formule comme ça plus qu'une } \\
\text { réalité. }\end{array}$ \\
\hline Personnalisé & $\begin{array}{l}\text { je t'envoie pleins de [smiley } \\
\text { coeur].Tu as l'air très } \\
\text { courageuse, tu vas t'en sortir et } \\
\text { montrer à cet élève que tu es plus } \\
\text { forte que lui. }\end{array}$ & $\begin{array}{l}\text { étre touchée, clest un petit témoignage, ça } \\
\text { ne fait pas avancer plus que ça, mais c'est } \\
\text { mignon.je me dis qu'il ne faut pas sucrer } \\
\text { tout le mignon, ça aide à voir un peu autre } \\
\text { chose, on se sent peut-être un peu moins } \\
\text { seule aussi }\end{array}$ \\
\hline
\end{tabular}

Tableau 2 : Exemples de réactions d'initiatrices à la suite de manifestations de soutien émotionnel stéréotypé et personnalisé

Table 2: Examples of initiators' reactions following stereotyped and personalized emotional support

Les éléments les plus appréciés du soutien par les participantes, à savoir le partage d'expérience (notamment émotionnel) et le soutien émotionnel personnalisé, sont teintés de la dimension émotionnelle. La prévalence de cette dimension laisse penser qu'elle s'observe également dans les échanges écrits. Les résultats de la recherche globale dans laquelle l'étude présentée ici s'inscrit (Prost, 2012) mettent effectivement en évidence une part explicite (ou manifeste) de cette dimension. Pour autant, tout un pan de celle-ci n'est pas visible sur les forums et est mis en évidence dans les entretiens, car elle relève de phénomènes de masquage et d'inférences des attentes de soutien psychologique. Les sections suivantes s'attachent à présenter ce type de résultats issus de la comparaison des émotions et attentes manifestes dans les échanges et exprimées en entretien. 


\section{2.- Masquages des émotions}

Notre analyse met en évidence des décalages entre les émotions communiquées par les participantes sur les forums et celles exprimées en entretien en référence à l'épisode relaté. Ces décalages prennent le plus souvent la forme de masquages, plus ou moins conscients, et de minimisation de l'état émotionnel ressenti sur le forum.

La comparaison entre les émotions manifestées dans les messages et celles exprimées durant les entretiens révèle que les participantes peuvent censurer certains de leurs états émotionnels tels que la honte, l'angoisse, le doute et le mal-être. Ce phénomène a été retrouvé chez quatre des neuf participantes interviewées (deux en position d'initiatrice et deux en position de réactante).

L'exemple d'Armelle illustre ce phénomène de masquage. Armelle réagit à la discussion initiée par Tara concernant l'agression dont elle a été victime. Elle ressent de la compassion pour l'initiatrice et veut lui témoigner du réconfort en faisant part d'une expérience similaire à laquelle elle a été confrontée peu de temps auparavant. Le tableau 3 montre les deux séquences qui ont servi à la mise en lumière du décalage (les éléments en gras renvoient aux expressions émotionnelles).

\begin{tabular}{|c|c|}
\hline Vécu exprimé dans le message écrit & Vécu exprimé en entretien \\
\hline $\begin{array}{l}\text { « [Tara], je te soutiens de tout mon cœur [smiley cœur] } \\
\text { Ce que tu racontes me touche beaucoup, car à une } \\
\text { échelle moindre, il m'est arrivé une chose similaire. Un } \\
\text { élève de ma classe s'est énervé et a voulu taper un de } \\
\text { ses camarades. j'ai voulu l'en empêcher et l'ai retenu } \\
\text { par-derrière, mais les coups ont fusé (...). J'ai été très } \\
\text { choquée (...) Moi personne ne m'a demandé comment } \\
\text { j'allais. Heureusement, } 2 \text { jours après c'était les } \\
\text { vacances, j'ai quand même eu le droit à des } \\
\text { anxiolytiques et (...) j'étais toute contracturée. Je } \\
\text { pense que la hiérarchie s'en fiche, ils ne sont pas sur le } \\
\text { terrain.» }\end{array}$ & $\begin{array}{l}\text { "Oui c'est exactement ça, je ne change rien. Je me } \\
\text { souviens que j'étais toute courbaturée, il avait } \\
\text { tellement de force, j'avais tellement honte qu'il } \\
\text { m'ait mis par terre. J'ai raconté le soir à mon copain, } \\
\text { j'avais vraiment honte (...) tout le monde nous } \\
\text { regardait et personne n'a été chercher quelqu'un (...) } \\
\text { ça m'a vraiment choqué.» }\end{array}$ \\
\hline
\end{tabular}

Tableau 3 : Mise en évidence de la censure de la honte

Table 3: Detection of the censure of shame and loneliness

Armelle fait part dans son message du choc qu'elle a ressenti après l'incident avec l'élève, elle donne des éléments sur son état psychologique («choquée », «anxiolytiques ») et sur son état physique (« contracturée »). Lors de l'entretien, elle considère ces propos conformes à ce qu'elle a ressenti. Toutefois, l'entretien apporte des éléments supplémentaires sur son état émotionnel : elle explique en entretien avoir ressenti de la honte (à deux reprises) lors de l'événement, émotion dont elle ne fait pas part à l'écrit. Cet exemple met en évidence le phénomène de masquage de l'état émotionnel vécu durant l'épisode relaté dans les messages.

L'analyse du contenu des échanges (voir Prost, 2012) montre que le partage d'expérience émotionnel prend une part importante des messages des initiatrices qui expriment des émotions liées à la tristesse et au mal-être (retrouvées dans 7 des 9 premiers messages des discussions que les participantes interrogées ont initiées), au doute (7/9), à la déception (4/9), à la colère (3/9), au stress (3/9), à la lassitude (3/9) et à la solitude (2/9). Pour autant, les participantes interviewées censurent certaines de leurs émotions telles que la honte, émotion souvent considérée comme difficilement communicable (Finkenauer, \& Rimé, 1998). Deux des quatre participantes expliquent, en entretien, masquer ou minimiser consciemment leur état émotionnel. Les motifs invoqués sont liés à de la pudeur de dévoiler l'intégralité de son ressenti émotionnel négatif «je ne dis pas que je suis à bout ou que je craque, je décris plutôt les faits, je ne me livre pas à propos de mon mal-être, je réserve ça à ma famille ».

Les phénomènes de masquage des émotions ne sont pas uniquement observés chez les initiatrices, mais le sont aussi chez les réactantes. Ceci est à mettre en lien avec le fait que les attentes des participants sont similaires qu'ils soient initiateurs ou réactants (Prost, Cahour, 
\& Détienne, soumis). Les participants, quel que soit leurs statuts, s'investissent dans une discussion dont le sujet fait écho à leur propre situation et tentent par ce biais de résoudre les éventuelles difficultés auxquelles ils sont confrontés. Ce phénomène de masquage permet de montrer que l'anonymat ne permet pas de dévoiler l'ensemble de ses émotions ressenties.

\section{3.- Masquage des attentes de soutien psychologique par les initiateurs}

Six des huit participantes interviewées en position d'initiatrice font part, en entretien, d'attentes différentes de celles exprimées dans leurs messages. Il n'y a pas de décalages observés pour les deux autres participantes, leurs attentes exprimées à l'écrit sont les mêmes que celles exprimées en entretien.

Le tableau 4 montre des exemples de décalages entre les attentes exprimées dans les messages et en entretien.

\begin{tabular}{|c|c|c|c|c|}
\hline \multirow[b]{2}{*}{ Interviewée } & \multicolumn{2}{|c|}{ Attentes exprimées à l'écrit } & \multicolumn{2}{|c|}{ Attentes exprimées en entretien } \\
\hline & $\begin{array}{l}\text { Nature de } \\
\text { l'attente }\end{array}$ & Extraits du message & $\begin{array}{l}\text { Nature de } \\
\text { l'attente }\end{array}$ & Verbatim \\
\hline Tara & Conseil & $\begin{array}{l}\text { Bref si quelques-uns } \\
\text { parmi vous ont des } \\
\text { conseils à me donner je } \\
\text { suis tout ouïe }\end{array}$ & $\begin{array}{l}\text { Décharge } \\
\text { émotionnelle } \\
\text { devant une } \\
\text { audience « vider } \\
\text { son sac » }\end{array}$ & $\begin{array}{l}\text { J'attendais pas plus de conseils que } \\
\text { ça (...) il fallait que je vide ça...En } \\
\text { l'écrivant c'était je me vide mon } \\
\text { sac, mais si une ou } 2 \text { personnes } \\
\text { avaient quelque chose à dire alors } \\
\text { je prenais. }\end{array}$ \\
\hline Jeanne & $\begin{array}{l}\text { Conseil } \\
\text { Information }\end{array}$ & $\begin{array}{l}\text { Quels sont les moyens de } \\
\text { pression face à des } \\
\text { enfants comme ça, qui } \\
\text { dépassent complètement } \\
\text { les limites; est-ce qu'il } \\
\text { existe encore des conseils } \\
\text { de discipline? Est-ce } \\
\text { qu'on peut envisager une } \\
\text { exclusion? }\end{array}$ & $\begin{array}{l}\text { Partage } \\
\text { d'expériences } \\
\text { similaires ; } \\
\text { Réassurance ; }\end{array}$ & $\begin{array}{l}\text { Ça a été difficile donc j'avais } \\
\text { besoin de soutien parce que je ne le } \\
\text { trouvais pas à l'école (...) des } \\
\text { personnes qui par exemple étaient } \\
\text { confrontées aux mêmes difficultés et } \\
\text { me rassurer, me dire que ça ne } \\
\text { venait pas forcément de moi et que } \\
\text { ma classe était vraiment difficile et } \\
\text { que c'était beaucoup de pas de bol }\end{array}$ \\
\hline Armelle & Conseil & $\begin{array}{l}\text { Que feriez-vous à ma } \\
\text { place? }\end{array}$ & $\begin{array}{l}\text { Soutien } \\
\text { émotionnel ; } \\
\text { Écoute }\end{array}$ & $\begin{array}{l}\text { les gens ne peuvent pas dire que tu } \\
\text { te plains quand tu demandes des } \\
\text { conseils. C'est pour ça que je } \\
\text { demande plus facilement des } \\
\text { conseils pratiques que du soutien } \\
\text { (...) j'ai toujours peur, dans la } \\
\text { vraie vie c'est pareil, j'ai toujours } \\
\text { peur qu'on me dise que je me plains } \\
\text { (...) des fois ça me manque, } \\
\text { jaimerais qu'on m'écoute et qu'on } \\
\text { me comprenne, mais je veux qu'on } \\
\text { me demande si on ne me demande } \\
\text { pas, je n'irais pas le dire }\end{array}$ \\
\hline
\end{tabular}

Tableau 4 : Exemples d'attentes exprimées pendant les discussions écrites confrontées aux attentes exprimées en entretien

Table 4: Examples of expectations expressed during written discussions compared to expectations expressed in interviews

Notons que les interviewées, en plus de masquer leurs émotions lors des échanges écrits, peuvent avoir masqué (volontairement ou non) certaines de leurs émotions lors de l'entretien.

Les attentes exprimées en entretien par les participantes sont de deux ordres : les attentes de soutien psychologique et le besoin de décharge émotionnelle devant une audience («vider son sac»). Nous considérons ce besoin de décharge comme une attente, car exprimer ses émotions devant une audience est un signe d'attente implicite.

La plupart des participantes expriment dans leur message initial une demande de conseil, de solution ou d'information. Le soutien psychologique attendu par les participantes peut se manifester par de la réassurance, de la compréhension, de l'écoute, des encouragements et des expressions de déculpabilisation (soutien émotionnel personnalisé) ou par du partage de 
situation similaire. Ceci renvoie aux éléments le plus appréciés du soutien mis en évidence précédemment.

Néanmoins, deux types de phénomènes sont aussi mis en œuvre plus ou moins consciemment $^{2}$ par les participantes au moment des discussions sur forum :

- Au lieu d'exprimer leur attente de soutien émotionnel et/ou leur besoin de décharge émotionnelle, elles expriment un autre type d'attente, le plus souvent des conseils (type de demande peut-être conforme à ce qui est attendu sur le forum) ;

- Elles masquent leur attente de soutien émotionnel et de décharge émotionnelle sans émettre une autre demande. L'attente de la participante est alors implicite. Dans quelle mesure, les réactants comprennent ce type d'attente et le cas échéant, sur quels indices ils se basent pour faire cette inférence?

Les attentes exprimées dans le premier message peuvent évoluer au fil des échanges et faire apparaître de nouvelles attentes, peu présentes au départ de la discussion. L'exemple de Lena, présenté dans le tableau 5, illustre ce phénomène qui est trouvé chez deux participantes. Lena exprime, dans son premier message, une difficulté à savoir quels contenus sont à enseigner aux élèves et demande aux participants de l'aider concrètement, via du partage d'expérience, pour savoir ce qu'elle doit mettre en place. Le premier réactant propose un type de soutien un peu en décalé de ce que Lena attendait. Il lui propose une réévaluation de la situation à la lumière de ses connaissances, ce qui provoque un autre type d'attente chez Lena :

\begin{tabular}{|c|c|}
\hline Message du premier réactant & Évaluation du message par Lena en entretien \\
\hline $\begin{array}{l}\text { «(..) il faut se fixer des priorités en fonction du } \\
\text { profil de ta classe, les programmes que nous avons à } \\
\text { suivre sont assez ambitieux par rapport au temps qui } \\
\text { nous est accordé pour les appliquer }(\ldots) »\end{array}$ & $\begin{array}{l}\text { "ça ça me conforte un peu dans mon idée, mais ce qui } \\
\text { me pose problème c'est que j'ai l'impression qu'il ne } \\
\text { faudrait pas faire ça, mais elle me dit que finalement } \\
\text { c'est ce qu'il faut faire » }\end{array}$ \\
\hline
\end{tabular}

Tableau 5 : Exemple d'évolution des attentes

Table 5: Example of evolving expectations

Lena explique en entretien que ce message l'a aidé à élaborer une nouvelle représentation de la situation qu'elle pressentait, mais qu'elle n'avait pas mentionnée dans son message, à savoir qu'elle ne peut suivre l'ensemble du programme scolaire et qu'elle doit faire des choix. Lena pressentait avant même d'aller sur le forum qu'elle ne pourrait faire l'intégralité de son programme, mais avait «l'impression qu'il ne faudrait pas faire ça ». La succession de messages analogues permet d'asseoir la nouvelle perspective de Lena «Le fait d'avoir tous ces messages (...) ce n'est pas que ça me fait oublier les préoccupations concrètes, mais je me dis : bon, on va faire au maximum et puis voilà ; je me sens déjà mieux ».

Cet exemple montre que les attentes évoluent au fil des échanges et que les réponses décalées peuvent permettre de répondre à des attentes moins prégnantes au départ. Dans l'exemple de Lena, la nouvelle évaluation de la situation lui permet de réduire son état émotionnel négatif sans que sa demande initiale soit assouvie.

\section{4.- Inférences des attentes de soutien psychologique de l'initiateur et projection de ses propres besoins par les réactants}

Les entretiens ont permis de mettre en évidence que les participantes, lorsqu'elles ont le statut de réactantes, tentent de comprendre les attentes réelles de l'initiateur afin de proposer un soutien adapté. Ce phénomène a été mis en évidence chez quatre des cinq réactantes. Elles infèrent les besoins d'après le style rédactionnel et le contenu des messages le besoin de soutien psychologique, attente non exprimée par les initiatrices.

2 Il est difficile d'évaluer dans quelle mesure les participantes sont conscientes ou pas de ces processus pendant la discussion écrite. 
Ce processus d'inférence peut être illustré avec l'exemple de la discussion "agressée et politique de l'autruche » pour laquelle nous avons réalisé un entretien avec l'initiatrice et deux réactantes. Rappelons que Tara émet une demande de conseils dans son premier message et exprime ses émotions tout au long de ses messages. En entretien, les deux réactantes infèrent l'attente de soutien émotionnel de Tara grâce à son dévoilement de soi. L'extrait du tableau 6 correspond à la séquence du message de l'initiatrice qui a permis à une réactante de faire cette inférence.

\begin{tabular}{|l|l|}
\hline Extrait du message de Tara & Inférence d'Armelle révélée en entretien \\
\hline $\begin{array}{l}\text { "Je me sens bien seule alors que j'attendais un } \\
\text { certain soutien de l'équipe de circonscription » }\end{array}$ & $\begin{array}{l}\text { "J'en conclus qu'elle dit « aidez-moi, } \\
\text { soutenez-moi »» }\end{array}$ \\
\hline
\end{tabular}

Tableau 6 : Exemple d'inférence des attentes grâce au dévoilement de soi

Table 6: Example of inference expectations through self-disclosure

Les participantes perçoivent chez l'initiatrice un besoin de soutien psychologique en se basant sur la forme de ses messages (dévoilement émotionnel) et le contenu « $\mathrm{j}$ 'attendais un soutien ». Sur le même message de Tara, une autre réactante commente en entretien « "C'est le genre de message où je n'arrive pas vraiment à identifier ce qu'elle demande (...) elle nous dit qu'elle se sent seule et qu'elle veut des conseils, mais des conseils je ne vois pas sur quoi (...) Je pense que ce qu'elle cherche c'est du soutien, mais du soutien de personnes qui font le même métier qu'elle et qui peuvent comprendre ou imaginer ce qu'elle est en train de vivre. »

Les entretiens ont mis en lumière que le soutien psychologique se manifeste de différentes façons selon les participantes. Les réactants font un effort de compréhension des attentes réelles de l'initiateur notamment en ce qui concerne les attentes de soutien émotionnel. Les exemples montrent que les réactantes projettent leurs propres attentes (passées ou présentes) pour élaborer le contenu de leur message qui a une fonction de soutien psychologique. Pour certaines, cela se traduit par du partage d'expérience similaire en ce qu'il permet de réduire le sentiment de solitude par la comparaison sociale. Armelle, qui est dans ce cas, explique en entretien "J'étais là, oh, mais la pauvre, je voulais lui montrer qu'elle n'était pas toute seule à vivre ce genre de situation. Pour moi c'est le vécu qui est important alors je lui fais part du mien ». Elle lui écrit un message dans la discussion où elle lui fait part d'un vécu similaire "[Tara], je te soutiens de tout mon cœur [smiley cœur]. Ce que tu racontes me touche beaucoup, car à une échelle moindre, il m'est arrivé une chose similaire (...)». Le soutien psychologique peut également passer par des marques explicites de soutien émotionnel. Lena explique en entretien avoir bénéficié de ce type de soutien sur le forum "Le fait d'avoir du soutien sans forcément des conseils, mais déjà de l'empathie, ça fait du bien (...) J'en ai beaucoup profité (...) Là l'idée c'est plus de faire comme les autres ont fait dans les autres discussions : ce que tu vis c'est dur et ce que tu fais c'est bien, essayer de la rassurer et de l'encourager »; elle témoigne à Tara de soutien psychologique à travers l'expression de soutien émotionnel personnalisé "(...) ce que tu as vécu est violent à tous points de vue et que je comprends ta détresse, ta colère, ton angoisse... (...) je te souhaite beaucoup de courage, j'espère que tu trouveras dans ton équipe de circo quelqu'un qui pourra entendre ce que tu as à dire! »

Ces exemples montrent que les réactantes infèrent les attentes de soutien psychologique des initiateurs à partir du dévoilement de soi émotionnel et du contenu du message. Elles vont ensuite manifester ce type de soutien à travers un mode de soutien qu'elles-mêmes attendent ou qui les a déjà aidées par le passé.

Le tableau 7 synthétise les résultats en montrant quel(s) phénomène(s) a (ont) été observé(s) sur quelles discussions. Notons que la discussion «élève qui n'aime pas l'école » n'a pas été gardée pour l'analyse puisque la participante n'a pas été en évocation lors de l'entretien (cf. 3.2. Méthodologie). 


\begin{tabular}{|c|c|c|c|c|}
\hline Sujet de la discussion & $\begin{array}{c}\text { Statut des } \\
\text { participantes } \\
\text { interviewées }\end{array}$ & $\begin{array}{l}\text { Masquage des } \\
\text { émotions } \\
\text { (initiatrice et } \\
\text { réactante) }\end{array}$ & $\begin{array}{l}\text { Masquage des } \\
\text { attentes de } \\
\text { soutien } \\
\text { psychologique } \\
\text { (initiatrice) }\end{array}$ & $\begin{array}{l}\text { Inférences des } \\
\text { attentes de } \\
\text { soutien } \\
\text { psychologique } \\
\text { (réactante) }\end{array}$ \\
\hline $\begin{array}{l}\text { Dysfonctionnement } \\
\text { institutionnel }\end{array}$ & Initiatrice & Non & Oui & - \\
\hline Méthode de lecture & Réactante & Non & _ & Non \\
\hline $\begin{array}{c}\text { Élève qui pleure tous } \\
\text { les matins }\end{array}$ & Réactante & Non & - & Oui \\
\hline \multirow{3}{*}{ Agression par un élève } & Initiatrice & Oui & Oui & - \\
\hline & Réactante & Oui & - & Oui \\
\hline & Réactante & Non & - & Oui \\
\hline $\begin{array}{l}\text { Gestion d'une classe } \\
\text { difficile }\end{array}$ & Initiatrice & Non & Oui & - \\
\hline $\begin{array}{l}\text { N'arrive pas à faire tout } \\
\text { son programme et ne } \\
\text { sait pas sur quoi insister }\end{array}$ & Initiatrice & Non & Oui & - \\
\hline Lassitude de son métier & Réactante & Oui & - & Oui \\
\hline $\begin{array}{l}\text { Problèmes relationnels } \\
\text { avec ses collègues et la } \\
\text { hiérarchie à cause de } \\
\text { ses absences }\end{array}$ & Initiatrice & Non & Oui & - \\
\hline $\begin{array}{l}\text { Gestion d'une classe } \\
\text { difficile }\end{array}$ & Initiatrice & Oui & Oui & - \\
\hline $\begin{array}{l}\text { Livret scolaire à } \\
\text { améliorer }\end{array}$ & Initiatrice & Non & Non & - \\
\hline
\end{tabular}

Tableau 7 : Synthèse des résultats

Table 7: Summary of the results

\section{5.- Discussion}

On observe que la dimension émotionnelle prend une place importante dans l'appréciation du soutien et que deux types de soutien teintés de cette dimension sont appréciés par l'ensemble des participantes. Le premier est le partage d'expérience, notamment émotionnel, qui est un gage de compréhension d'autrui et d'un soutien de qualité. Ainsi, au regard de nos résultats, le modèle du «bon partenaire» proposé par Christophe et Di Giacomo (2003) semble pouvoir être réexaminé. Selon ces auteurs, le bon partenaire doit éviter de parler de ses propres problèmes. Au contraire, notre étude montre que de nombreux participants au forum cherchent à se rassurer par le constat de ne pas être seuls dans la situation difficile. Le second type de soutien qui est particulièrement apprécié est le soutien émotionnel personnalisé. Il se traduit par de l'écoute, de la compréhension, de la réassurance, des encouragements ou de la déculpabilisation. Il constitue une autre attente partagée par toutes les interviewées quand elles sont en position d'initiatrices.

Bien que la dimension émotionnelle ait une place centrale dans la satisfaction du soutien, nos résultats ont montré que les participantes (initiatrices et réactantes) peuvent masquer, plus ou moins consciemment, dans les échanges écrits certaines de leurs émotions comme la honte, la culpabilité ou le mal-être. Outre le masquage de ces émotions, notre étude a montré que 
les besoins de décharge émotionnelle et de soutien psychologique sont systématiquement masqués à l'écrit par les initiatrices. L'analyse des entretiens a permis de mettre en évidence deux formats de dévoilement des attentes de soutien psychologique plus ou moins conscients et masqués, d'où la complexité de leur approche et de l'intérêt de croiser les méthodes. (1) les attentes explicites, socialement partagées dans les messages écrits et en entretien. Il s'agit le plus souvent de demande de conseils et de partage d'expérience (Prost, 2012). Aucun masquage, conscient ou non, n'est opéré dans ce cas ; (2) les attentes masquées pendant l'interaction sur le forum, mais explicitées en entretien. Un troisième format de masquage peut être envisagé : celui d'un masquage à tous (à l'écrit et en entretien). Le soutien psychologique attendu par les participantes peut se manifester par du soutien émotionnel personnalisé ou par du partage de situation similaire, ce qui renvoie aux éléments le plus appréciés du soutien. Certaines initiatrices substituent à cette demande une demande explicite de conseils ou d'informations alors que d'autres n'émettent explicitement aucune demande dans leur message. Cependant, ces attentes sont souvent comprises par les réactantes à partir d'indices qui leur permettent de faire l'inférence de cette attente. Les réactantes ont explicitement expliqué en entretien se baser sur le dévoilement de soi émotionnel et sur le contenu du message pour comprendre les attentes «réelles » des initiatrices. Elles vont alors manifester ce type de soutien à travers un mode de soutien qu'elles-mêmes attendent ou qui les a déjà aidées par le passé. L'étude a également montré que les attentes évoluent au cours des interactions et les réponses un peu décalées peuvent répondre à des besoins moins clairs au départ. Ainsi, les réponses apportées aident à définir le besoin et l'échange relève alors d'une co-construction.

Notre étude apporte un éclairage nouveau tant sur la manifestation émotionnelle que sur la compréhension des émotions sur les forums d'échanges entre pairs. Nos résultats contredisent la théorie de la réduction des indices sociaux en ce qu'ils mettent en évidence l'importance de la dimension émotionnelle dans les échanges entre professionnels sur les forums. En ce sens, notre étude alimente à l'inverse la théorie de la communication hyperpersonnelle proposée par Walther (1996). Toutefois, le masquage de certaines émotions et des besoins liés à la décharge émotionnelle et au soutien psychologique présenté dans cet article tend à nuancer la perspective de Walther (ibid). Ces phénomènes de masquage montrent que l'anonymat ne permet pas de dévoiler l'ensemble de ses émotions ressenties. Le dévoilement émotionnel sur les forums est relatif, même en situation médiatisée où l'anonymat et la distance physique pourraient envisager un dévoilement total comme l'envisage la théorie de la communication hyperpersonnelle. Les participants peuvent masquer ou minimiser, plus ou moins consciemment, certaines de leurs émotions, telles que la honte, le mal-être ou les doutes. Ce résultat étaye les conclusions de Finkenauer et Rimé (1998) qui montrent que la honte et la culpabilité échappent très souvent au partage social. Le cadre établi en entretien apparaît plus favorable à des dévoilements émotionnels, peut-être que la confiance instaurée permet aux participantes de livrer des états émotionnels difficilement partageables. Pour autant, nous ne pouvons pas assurer que les participantes n’ont pas également masqué certaines émotions lors de l'entretien.

Le masquage des attentes exercé par les participants dans les échanges écrits peut se comprendre par le fait que les individus, comme le souligne Cahour (2010), cherchent à gérer la situation émotionnelle dans le sens socialement attendu. Or, les stratégies de coping centrées «problème» sont davantage valorisées que les stratégies d'ajustement centrées «émotions» ou centrées «soutien social» (Ponnelle, \& Lancry, 2002), d'où la nécessité pour certains participants de dissimuler leurs attentes de soutien psychologique en attentes de conseil, socialement plus valorisées. Ainsi, nous pensons que les règles sociales en vigueur ne permettent pas aux individus de demander explicitement du soutien psychologique. Les participants utilisent alors une voie détournée, celle de la communication implicite, pour exprimer ce type de besoin.

Le processus d'inférence du réactant concerne les attentes de soutien psychologique et 
repose sur la communication implicite. Le dévoilement émotionnel semble être une stratégie socialement reconnue de recherche d'empathie, ce qui va dans le sens de Hess et Kirouac (2000) pour qui une des fonctions du partage social des émotions est de susciter des réactions chez son interlocuteur. Nos résultats vont dans le sens de ceux présentés par Preece (1999) et Pfeil et Zaphiris (2007) en ce qu'ils mettent en évidence le masquage des attentes de soutien psychologique. Toutefois, les méthodes utilisées sont différentes, ces auteurs se positionnent d'un point de vue «extrinsèque » (Theureau, 1992) en se centrant sur l'analyse des échanges écrits. Ces auteurs montrent la prégnance du discours empathique et l'absence de demandes de soutien émotionnel, et en infèrent le masquage de ce type de soutien. Notre étude apporte un éclairage complémentaire en ce qu'elle se place de point de vue « intrinsèque » (Theureau, ibid.) des participants. Elle a montré le masquage des initiateurs et le processus d'inférence des réactants.

L'étude présentée ici apporte des connaissances supplémentaires sur la manifestation et la compréhension des émotions des participants à des forums virtuels. Ce travail initié sur un échantillon restreint de discussions et de participants nécessite d'être prolongé en continuant de se centrer sur le point de vue intrinsèque des participants. Dans notre étude, les participants aux forums sont des professionnels, type de communautés peu étudié par les études qui s'intéressent à la communication médiatisée sur les forums. Il convient ainsi de s'interroger sur les spécificités de cette population et du rôle du référentiel commun dans la compréhension d'une part, des situations professionnelles exposées, et d'autre part, dans la compréhension des manifestations émotionnelles.

\section{BIBLIOGRAPHIE}

Babinski, L. M., Jones, B. D., \& DeWert, M. H. (2001). The Roles of Facilitators and Peers in an Online Support Community for First-Year Teachers. Journal of educational and psychological consultation, 12(2), 151-169.

Barak, A. \& Gluck-Ofri, O., (2007). Degree and Reciprocity of Self-Disclosure in Online Forums. Cyberpsychology \& Behavior, 10(3), 407-417.

Cahour, B. (2000). Accès aux émotions censurées lors d'interactions agents-clients. In C. Plantin, M. Doury, \& V. Traverso (Eds.), Les émotions dans les interactions (pp. 109-117). Lyon: Presses Universitaires de Lyon.

Cahour, B. (2010). Emotions, affects et confort comme nouveaux déterminants de l'activité et de l'usage. In G. Valléry, M. Zouinar, M.C. Leport (Eds.). Ergonomie, conception de produits et services médiatisés (pp. ). Paris: PUF.

Cahour, B. (2012). Les émotions vécues, constitutives de l'activité. Cas des interactions de travail et des usages situés. Habilitation à Diriger des Recherches, Amiens.

Cahour, B., Brassac, C., Vermersch, P., Bouraouis, J. L., Pachoud, B., \& Salembier, P. (2007). Étude de l'expérience du sujet pour l'évaluation de nouvelles technologies : l'exemple d'une communication médiée. Revue d'anthropologie des connaissances, 1(1), 85-120.

Caplan, S., \& Turner, J. (2007) Bringing theory to research on comuter-mediated conforting communication. Computers in human behavior, 23(2), 985-998

Chanier, T., \& Cartier, J. (2006). Communauté d'apprentissage et communauté de pratique en ligne : le processus réflexif dans la formation des formateurs. Revue internationale des technologies en pédagogie universitaire, 3(3), 64-82.

Christophe, V., \& Di Giacomo, JP. (2003). Est-il toujours bénéfique de partager ses expériences émotionnelles? Rôle du partenaire dans les situations de partage social des émotions. Revue Internationale de Psychologie Sociale, 16(2), 99-124.

Cosnier, J. (1994). Psychologie des émotions et des sentiments. Retz: Paris.

Dejours, C. (1993). Travail : usure mentale, De la psychopathologie à la psychodynamique du travail, Nouvelle édition augmentée. Paris: Bayard.

Dejours, C. (1998). Souffrance en France. La banalisation de l'injustice sociale. Paris: Le Seuil.

Derks, D., Fischer, A. H., \& Bos, A. E. R. (2007). The role of emotion in computer-mediated 
communication: A review. Computers in Human Behavior, 24(3), 766-785.

Finkenauer, C., \& Rimé, B. (1998). Socially shared emotional experiences vs. emotional experiences kept secret: Differential characteristics and consequences. Journal of Social and Clinical Psychology, 17(3), 295-318.

Fisher-Lokou, J., Guéguen, N., \& Lépy, N. (2004). Effets de la communication par réseaux informatiques versus en face-à-face sur la représentation réciproque des négociateurs. Bulletin de Psychologie, 57(5), 525-533.

Gauducheau, N. (2008). La communication des émotions dans les échanges médiatisés par ordinateur : bilan et perspectives. Bulletin de Psychologie, 61(4), 389-404.

Goffman, E. (1974). Les rites d'interaction. Paris: Éditions de Minuit.

Grosjean, V., \& Ribert-Van de Weerdt, C. (2005). Vers une psychologie ergonomique du bien-être et des émotions : les effets du contrôle dans les centres d'appel. Le Travail Humain, 68(1-4), 355.

Hebert, B. \& Vorauer, J. (2003). Seeing througt the screen : is evaluative feedback communicated more effectively in face-to-face or computer-mediated exchanges ? Computers in human behavior, 19(1), 25-38.

Helgeson, V. S. \& Gottlieb, B. H. (2000). Support groups. In S. Cohen, L. G. Underwood, \& B. H. Gottlieb (Eds.), Social support measurement and intervention: A guide for health and social scientist (pp. 221-245). New York: Oxford University Press.

Hess, U., \& Kirouac, G. (2000). Emotion expression in groups. In M., Lewis \& J., Haviland-Jones (Eds.), Handbook of emotions (pp. 368-381). New York: Guilford Press.

Hian, L. B., Chuan, S. L., \& Trevor, T. M. K. (2004). Getting to know you: exploring the development of relational intimacy in CMC. Journal of computer-mediated communication, 9(3).

Jiang, L., Bazarova, N. N., \& Hancock, J. T. (2011). The Disclosure-Intimacy Link in ComputerMediated Communication: An Attributional Extension of the Hyperpersonal Model. Human Communication Research, 37(1), 58-77.

Kato, Y., Kato, S., \& Akahori, K. (2007). Effects of emotional cues transmitted in e-mail communication on the emotions experiences by senders and receivers. Computers in Human Behavior, 23(4), 1894-1905.

Kraut, R., Patterson, M., Lundmark, V., Kiesler, S., Mukophadhyay, T., \& Scherlis, W. (1998). Internet paradox: A social technology that reduces social involvement and psychological wellbeing? American psychologist, 53(9), 1017.

Kruger, J., \& Epley, N. (2005). Egocentrism over the email: can we communicate as well we think ? Journal of personality and social psychology, 89, 925-936.

Lewkowicz, M., Marcoccia, M., Atifi, H., Bénel, A., Gaglio, G., Gauducheau, N., \& Tixier, M. (2008). Online Social Support: Benefits of an Interdisciplinary Approach for Studying and Designing Cooperative Computer-Mediated Solutions. Proceedings of the 8th Conference on the Design of Cooperative Systems, pp. 99-110.

Lipiansky, E. M. (1992). Identité et communication : l'expérience groupale. Paris: PUF.

Malik, S. H., \& Coulson, N. S. (2008). Computer-mediated infertility support groups: an exploratory study of online experiences. Patient Education and Counseling, 73(1), 105-113.

Marc, J., Grosjean, V., \& Marsella, C. (2011). Dynamique cognitive et risques psychosociaux : isolement et sentiment d'isolement au travail. Le travail humain, 74(2), 107-130.

Marcoccia, M., \& Gauducheau, N. (2007). L'analyse du rôle des smileys en production et en réception : un retour sur la question de l'oralité des écrits numériques. Glottopol, 10, 38-55.

Moody, E.J. (2001). Internet Use and Its Relationship to Loneliness. Cyberpsychology \& Behavior, $4(3), 393-401$.

Pfeil, U., \& Zaphiris, P. (2007). Patterns of Empathy in Online Communication. Présenté à CHI 2007 - Emotion \& Empathy, San Jose, CA, USA.

Pfeil, U., Zaphiris, P., \& Wilson, S. (2009). Older adults' perceptions and experiences of online social support. Interacting with computers, 21, 159-172.

Ponnelle, S. \& Lancry, A. (2002). Stratégies d'ajustement et ressources environnementales et personnelles dans la dynamique du stress. In M. Neboit \& M. Vézina (Eds.). Stress au travail et santé psychique (pp. 59-77). Toulouse: Octarès. 
Preece, J. (1999). Empathic communities: Balancing emotional and factual communication. Interacting with computers, 12(1), 63-77.

Preece, J. \& Ghozati, K. (2001). Observations and Explorations of Empathy Online. In. R. R. Rice \& J. E. Katz, The Internet and Health Communication: Experience and Expectations (pp. 237-260). Sage Publications Inc.: Thousand Oaks.

Prost, M. (2012). Les échanges entre professionnels de l'éducation : entre soutien psychologique et acquisition de connaissances sur la pratique. Thèse de doctorat, Paris, ENST.

Prost, M., Cahour, B., \& Détienne, F. (soumis). Le soutien psychologique et l'acquisition de connaissances sur la pratique : moteurs des échanges entre professionnels sur les forums. Le Travail Humain.

Proulx, S., \& Latzko-Toth, G. (2000). La virtualité comme catégorie pour penser le social : 1'usage de la notion de communauté virtuelle. Sociologie et sociétés, 32(2), 99-122.

Rimé, B. (2005). Le partage social des émotions. Paris: Presses Universitaires de France.

Sproull, L., \& Kiesler, S. (1986). Reducing social context cues: electronic mail in organizational communication. Management science, 32(11), 1492-1512

Theureau, J. (1992). Le cours d'action : analyse sémio-logique. Essai d'une anthropologie cognitive située. Berne: Peter Lang.

Tichon, J. G., \& Shapiro, M. (2003). The process of sharing social support in cyberspace. CyberPsychology \& Behavior, 6(2), 161-170.

Vermersch, P. (1994). L'entretien d'explicitation. Paris: ESF.

Wagenaar, W. A. (1986). My memory: A study of autobiographical memory over six years. Cognitive psychology, 18(2), 225-252.

Walther, J. (1996). Computer-mediated communication: impersonal, interpersonal and hyperpersonal interaction. Communication research, 23(1), p3-43.

Walther, J., \& Boyd, S. (2002). Attraction to computer-mediated social support. In Communication technology and society: audience adoption and uses, C.A. Lin et D.J. Atkin, Hampton Press: Creekill, pp. 153-188.

Walther, J., \& D'Addario, K. (2001). The impact of emoticons on messages interpretation in computer-mediated communication. Social science computer review, 19(3), 324-347.

Wenger, E. (1998). Communities of Practice: Learning, Meaning and Identity. New York: Cambridge University Press.

Wright, K.B. (2000). The communication of social support within an on-line community for older adults: a qualitative analysis of the SeniorNet community. Qualitative Research Reports in Communication, 1(2), 33-43.

Wright, K. B., \& Bell, S. B. (2003). Health-related support groups on the Internet: Linking empirical findings to social support and computer-mediated communication theory. Journal of Health Psychology, 8(1), 39.

Zhang, J., Qu, Y., Cody, J., \& Yuling, W. (2010). A case study of micro_blogging in the enterprise: use, value, and related issue. Présenté à CHI 2010 : Organizations and communities, Atlanta, USA, pp. 123-132.

\section{RESUME}

L'article s'intéresse au bien-être au travail et cherche à savoir si les forums sont un nouvel outil pour l'améliorer. L'étude présentée explore le vécu subjectif de participants à des forums de discussions entre professionnels de l'éducation. Il se centre sur les attentes et l'état émotionnel des participants. L'enjeu est de mettre en évidence les décalages entre ce qui est socialement partagé dans les échanges écrits et ce qui de l'ordre de l'intime, exprimé en entretien. Pour ce faire, des entretiens d'auto-confrontation avec des relances d'entretien d'explicitation basés sur les traces écrites de discussion ont été réalisés auprès de neuf participantes au forum. 
Les résultats montrent que le partage d'expérience, notamment émotionnel, et le soutien émotionnel personnalisé sont les deux types de soutien les plus attendus et les plus appréciés. La dimension émotionnelle est ainsi très présente dans la satisfaction $\mathrm{du}$ soutien. Pour autant, dans les échanges écrits, certaines émotions telles que la honte, la culpabilité et le mal-être ne sont pas communiquées. Le besoin de décharge émotionnel et de soutien psychologique sont des attentes systématiquement masquées dans les messages écrits, et parfois transformées en demande de conseils. On observe que les réactants infèrent les attentes de soutien psychologique de l'initiateur grâce au dévoilement de soi émotionnel. Ils vont ensuite manifester ce type de soutien à travers un mode de soutien qu'eux-mêmes attendent ou qui les a déjà aidés par le passé. Les résultats sont discutés au regard de la théorie de la communication hyperpersonnelle proposée par Walther (1996).

\section{MOTS CLEFS}

forums de discussion professionnels, soutien social, entretien d'auto-confrontation ; explicitation, masquage, émotions, attentes, inférence.

\section{REFERENCEMENT}

Prost, M., Cahour, B., \& Détienne, F. (2013). Masquage des émotions et des attentes de soutien psychologique sur des forums d'entraide professionnelle. Activités, 10(2), 20-38, http://www.activites.org/v10n2/v10n2.pdf

Article soumis le 23 janvier 2013, accepté pour publication le 6 juillet 2013 